\title{
Rectipilus stromatoides sp. nov. (Agaricales, Basidiomycota), a new cyphelloid fungus from the Brazilian Amazon
}

\author{
Gorjón SP ${ }^{1}$ and de Jesus MA ${ }^{2}$ \\ ${ }^{1}$ Universidad de Salamanca. Salamanca, España 37007. \\ ${ }^{2}$ Laboratório de Patologia da Madeira. Instituto Nacional de Pesquisas da Amazônia. Manaus, Brazil 69060-001
}

Gorjón SP, de Jesus MA 2014 - Rectipilus stromatoides sp. nov. (Agaricales, Basidiomycota), a new cyphelloid fungus from the Brazilian Amazon. Mycosphere 5(2), 393-396, Doi 10.5943/mycosphere/5/2/12

\begin{abstract}
A new cyphelloid species, Rectipilus stromatoides is described from the Brazilian Amazon. It is characterized by the basidiomes disposed in a pinkish stromatic structure and microscopically by the presence of cystidiols and the size of the basidiospores. The species is compared with the similar species. A key to the accepted species of Rectipilus is provided.
\end{abstract}

Key words - Brazil - corticioids - South America - tropical rainforest

\section{Introduction}

Rectipilus Agerer is a cyphelloid genus with currently nine species (Agerer 1973, 1979, Cooke 1989, Mycobank 2014, Vila et al. 1999). It is morphologically and phylogenetically very closely related to Henningsomyces Kuntze, producing similar, more or less tubular basidiomes. Henningsomyces produces somewhat gelatinous basidiomes (Agerer 1983) but the most important anatomical character to separate the two genera is the branching pattern of the surface hyphae. All species of Henningsomyces produce consistently branched surface hyphae, whereas those of Rectipilus species are usually non-ramified, a feature which is considered taxonomically important at the generic level among cyphelloid fungi (Agerer 1973, 1983, Bodensteiner et al. 2004). Both, Rectipilus and Henningsomyces have non-pigmented and non-encrusted surface hyphae, a character that morphologically separates them from a large number of cyphelloid genera (Agerer 1983). The phylogenetic study by Bodensteiner et al. (2004) suggests that there are two clades, each including a mixture of species of Rectipilus and Henningsomyces, so the inclusion of more species in the analyses is still required to elucidate their phylogenetic relationships

In April 2012, the senior author was invited to the "Instituto Nacional de Pesquisas da Amazônia" (INPA, Manaus, Brazil) to examine some collections of corticioid fungi from the Amazonas and Roraima states of Brazil. A deviating specimen is proposed as new species in Rectipilus based on morphological characters.

\section{Materials \& Methods}

\section{Macro- and microscopic examinations}

For light microscopy studies, samples were mounted in $3 \%$ potassium hydroxide $(\mathrm{KOH})$, 
Melzer's reagent (IKI). A solution of $0.1 \%$ cotton blue in $60 \%$ lactic acid (CB) was used to determine cyanophily of basidiospore walls. Line drawings were made with a camera lucida attachment. All the specimens are deposited in INPA.

Rectipilus stromatoides Gorjón, sp. nov.

Figs $1-6$ MycoBank 808176

Diagnosis - It differs from other species in the genus by the diverse basidiome disposition in a stromatic structure. It is microscopically similar to Rectipilus erubescens, this with larger basidiospores and to $R$. natalensis, the latter with smaller basidiospores.

Type -Brazil, Amazonas, Manaus, Reserva florestal A. Ducke, 27 Apr 2008, on unidentified hardwood trunk, coll. M.A. Jesus, LPS 3536 (INPA, holotype).

Etymology - stromatoides: referred to the disposition of the basidiomes in a stroma.

Description - Basidiomes tubular, often with a median constriction, usually 200-300(-600) $\mu \mathrm{m}$ long and about $100-150 \mu \mathrm{m}$ in diam., arranged and embedded in a dense, and largely effused, vivid pink coloured stroma. The external part of the basidiomes are composed by a mantle of densely interwoven hyphae, some of them hanging loosely or in disorder and projecting, usually tortuose or spirally curved, not encrusted nor pigmented. This external layer is about 15-20 $\mu \mathrm{m}$ wide in transverse section. To the internal part of the basidiome the hyphae are more loosely arranged producing the hymenium. Pore not very patent, formed by convulated hyphae in the apical part. All hyphae with clamps, thin-walled or with distinct walls, but not distinctly thick-walled, hyaline, not encrusted in $\mathrm{CB}, \mathrm{KOH}$, Melzer's reagent, or water, not reacting in Melzer's reagent. Hymenium a palisade of basidia and cystidiols. Basidia clavate, 18-30 × 6-10 $\mu \mathrm{m}$, with four slender sterigmata, and a basal clamp. Cystidiols clavate to cylindrical, and usually with a more or less elongated apical papilla, about the same size of the basidia. Basidiospores broadly ellipsoid, 5$5.5(-6) \times 3-3.5 \mu \mathrm{m}$, smooth, with firm to thickened walls, IKI-, CB-.

Known distribution - Known only from one collection in the type locality in the central Amazon basin.

Other material examined $-R$. erubescens: N-Rhodesien, Lubalansuki Hill, Namwala, B. L. Mitchel, 7 May 1964, on Vellozia equisedoides, (K 190550, holotype).

\section{Discussion}

As indicated above, species of Rectipilus produce small tubiform basidiomes with smooth, non-pigmented, and non-branched surface external hyphae. Infrageneric characters contemplate reaction of the hyphae in Melzer's reagent, shape of the surface hyphae, shape and size of the basidiospores, shape, size and colour of the basidiomes, and presence of a more or less developed subiculum. So far, nine species are accepted in Rectipilus: $R$. bavaricus Agerer, R. cistophilus Estreve-Rav. \& Vila, R. confertus (Burt) Agerer, $R$. davidii (D.A. Reid) Agerer, $R$. erubescens (D.A. Reid) Agerer, R. fasciculatus (Pers.) Agerer, R. idahoensis (W.B. Cooke) Agerer, and $R$. natalensis (W.B. Cooke) Agerer. There is an additional species listed in Rectipilus (Mycobank 2014), R. sulphureus (Sacc. \& Ellis) W.B. Cooke that should be excluded from this genus because according to the descriptions (Cunningham 1963: 311) the surface hyphae are finely encrusted and pigmented.

Rectipilus stromatoides is morphologically similar to Rectipilus natalensis from South Africa, by the tubiform basidiomes and spirally curved external hyphae but the latter differs in larger yellowish basidiomes, absence of cystidiols, and smaller, thin-walled basidiospores $(3.5-4.5 \times 2.5-$ $3.5 \mu \mathrm{m})$ (Agerer 1973). Rectipilus stromatoides also resembles Rectipilus erubescens, described from Zambia, sharing the pale rose colour, but this species usually has less tortuose external hyphae, lacks cystidiols, and has larger basidiospores $(6-7.5 \times(4-) 4.5-5.5(-6) \mu \mathrm{m})$ (Agerer 1979). Based on the examination of the holotype of $R$. erubescens, the basidiospores have firm to somewhat thickened-walls. In addition, the basidiome disposition in a conspicuous stroma is a unique character of $R$. stromatoides. 


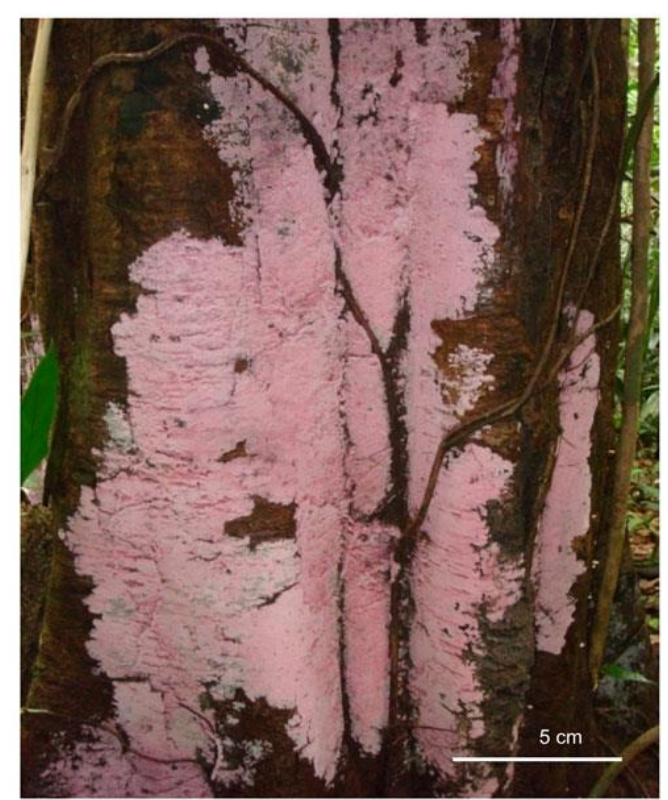

1

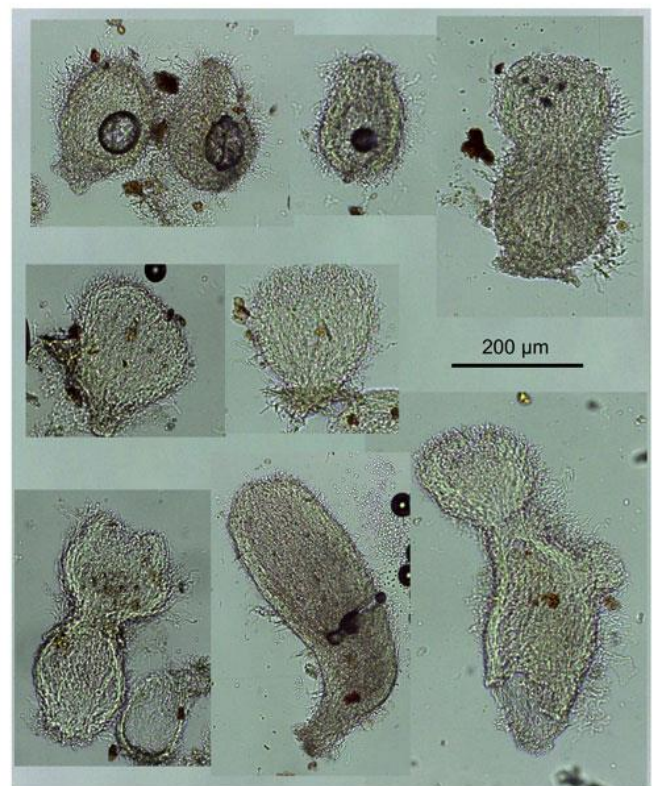

2

Figs 1-2-Rectipilus stromatoides. 1, Aspect of the aggregate basidiomes in the stroma. 2, Details of individual basidiomes.
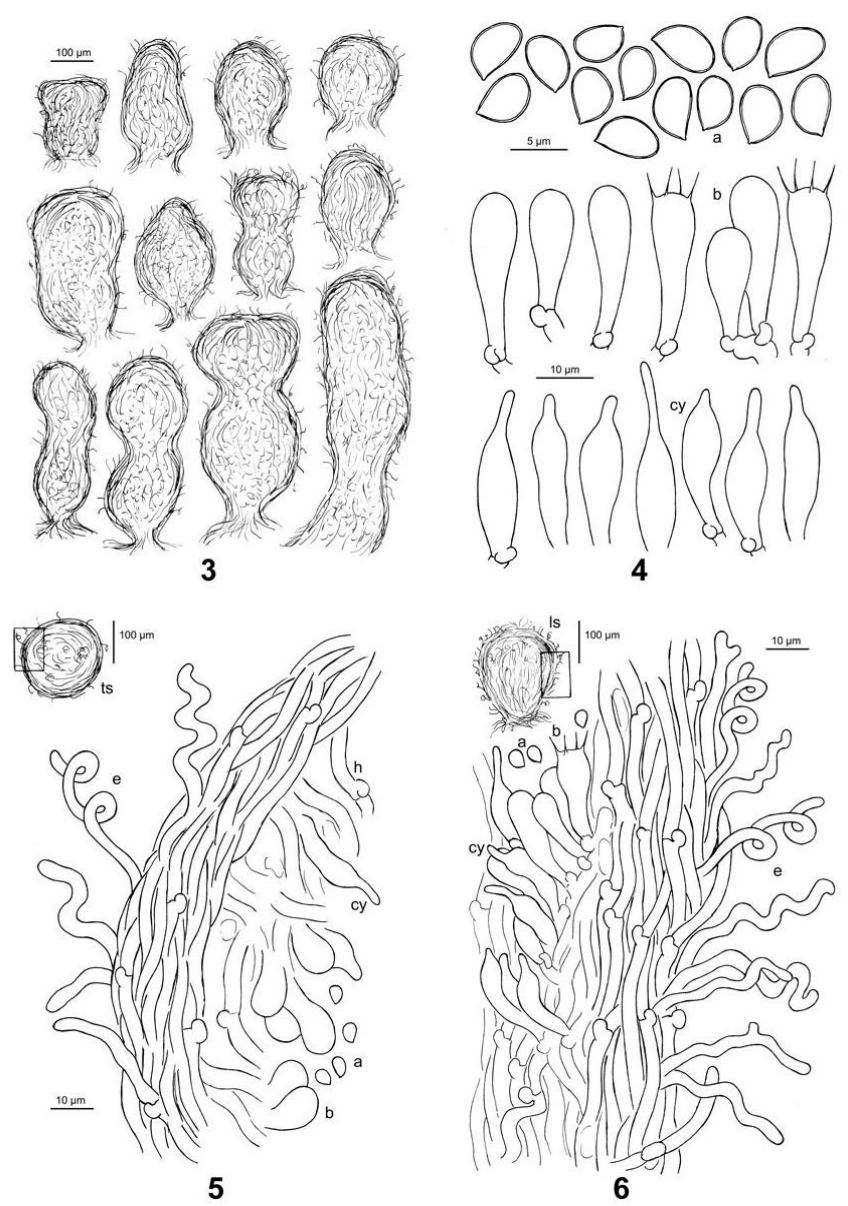

Figs 3-6 - Rectipilus stromatoides. 3, Basidiomes. 4, Basidiospores, basidia and cystidiols. 5, Transversal section of the basidiome. 6, Longitudinal section of the basidiome. a. Basidiospores. b. Basidia. cy. Cystidiols. e. Hyphae (external hyphae of the basidiome). h. Hyphae (generative). ls. Longitudinal section of the basidiome. ts. Transverse section. 


\section{Key to Rectipilus species}

1. Surface hyphae of the basidiome thick-walled and dextrinoid................................................ 2

1. Surface hyphae not dextrinoid . 3

2. Basidiospores ellipsoid to ovoid, $4-5.5 \times 3-5 \mu \mathrm{m}$ R. idahoensis

2. Basidiospores subglobose, $6.5-7.5 \times 6-7 \mu \mathrm{m}$ R. davidii

3. Surface hyphae more or less straight

3. Surface hyphae tortuose

4. Basidiomes shortly tubiform, up to $0.3 \mathrm{~mm}$ long R. bavaricus

4. Basidiomes longer, ab. 1-2.5 mm long 5

5. Basidiospores 4.5-6(-7.5) $\times 2.5-3.5 \mu \mathrm{m}$, subiculum well developed R. confertus

5. Basidiospores (5-)5.5-7 $\times 3.5-5 \mu \mathrm{m}$, subiculum absent or little developed.

6. Basidiomes disposed in a stromatic structure

6. Basidiomes aggregated but not embedded in a stroma.

R. stromatoides

7. Basidiomes with a pale rose colour, basidiospores 6-7.5 $\mu \mathrm{m}$ long. R. erubescens

7. Basidiomes with a whitish or yellowish colour, basidiospores up to $5.5 \mu \mathrm{m}$ long 8

8. Basidiomes ab. 0.5-1 mm long, basidiospores 3.5-4.5 $\mu \mathrm{m}$ long R. natalensis

8. Basidiomes ab. 0.2-0.6 mm long, basidiospores slightly longer, 4-5.5 $\mu \mathrm{m}$ long R. cistophilus

\section{Acknowledgements}

Travel and residence expenses of Sergio P. Gorjón were covered by the Brazilian government during the visiting period through the "Conselho Nacional de Desenvolvimento Científico e Tecnológico" (CNPq). Francisco Javier Hernández García and Bryn Dentinger, curators of SALA and KEW respectively, managed the loan of $R$. erubescens. The critical review and suggestions of an anonymous reviewer is sincerely appreciated.

\section{References}

Agerer R. 1973 - Rectipilus, eine neue Gattung cyphelloider Pilze. Persoonia 7, 389-436

Agerer R. 1979 - Typusstudien an cyphelloiden Pilzen II. Rectipilus erubescens. Sydowia 32, 1-4.

Agerer R. 1983 - Typus studien an cyphelloiden Pilzen IV. Lachnella Fr. s.l. Mitteilungen aus der Botanischen Staatssammlung München 19, 163-334.

Bodensteiner P, Binder M, Agerer R, Moncalvo JM, Hibbett DS. 2004 - Phylogenetic diversity of cyphelloid forms in the homobasidiomycetes. Molecular Phylogenetics and Evolution 33, 501-515.

Cooke WB. 1989 - The cyphelloid fungi of Ohio. Memoirs of the New York Botanical Garden 49, $158-172$.

Cunningham GH. 1963 - The Thelophoraceae of Australia and New Zealand. Bulletin of the New Zealand Department of Industrial Research 145, 1-359.

Vila J, Esteve-Raventós F, Llimona X. 1999 - Rectipilus cistophilus Esteve-Rav. et Vila sp. nov., un nuevo hongo cifeloide mediterraneo. Revista catalana de micología 22, 1-4.

www.mycobank.org - 2014 . 